\title{
The effects of mode on interpreting performance in a simulated police interview
}

Sandra Hale, Jane Goodman-Delahunty, Natalie Martschuk, Stephen Doherty

\section{Abstract}

This study tested the effects of the consecutive and simultaneous interpreting modes in a simulated police interview, addressing four research questions: (1) Does the consecutive interpreting mode lead to more accurate interpreting than the simultaneous interpreting mode? (2) Do language combinations moderate the performance of similarly qualified interpreters? (3) Does experience in simultaneous interpreting in legal settings increase interpreting accuracy in SI? and (4) Which mode of interpreting do interpreters perceive to require more mental effort? A total of 70 interpreters interpreted a live simulated interview between an English-speaking interviewer and an Arabic-, Mandarin- or Spanish-speaking suspect. Mode was varied within participants, and the order of the mode was counterbalanced across participants. Interpreters rated their perceived mental effort after the task. Independent assessments of performance showed better results for the simultaneous interpreting mode, regardless of language. This effect held for accuracy of style, verbal rapport markers, and interpreting protocol.

Key words: interpreting mode, simultaneous, consecutive, legal interpreting 


\section{Introduction}

Modes of interpreting are commonly associated with particular settings: simultaneous is the default mode for international conference settings and short consecutive is the default mode for domestic settings (Pöchhacker 2011b; Russell and Takeda 2015). This association has generally been due to the nature of the settings. Conference interpreting settings are typically formal and monologic, where interpreters sit at a distance from the speakers in a booth with simultaneous interpreting equipment, interpreting speeches unidirectionally (generally from language B to language A). Domestic settings, on the other hand, are mostly dialogic and less formal, with the interpreter present in the interaction, interpreting each turn from each of the speakers bidirectionally (from and into languages A and B). There are exceptions to these trends. The classic long consecutive mode is still used in some conferences, primarily dinner speeches and the like (Pöchhacker 2004; Seeber 2015). Simultaneous interpreting is used in some community interpreting settings such as courts and mental health sessions, often without equipment, in what is called the whispering or chuchotage mode (Hale 2007). These two types of instances are also monologic. For example, when a witness is giving evidence in the language of the court, the interpreter sits next to the accused, interpreting simultaneously into the accused's language to make them linguistically present in their own proceedings (Judicial Council on Cultural Diversity 2017). In mental health settings, interpreters interpret simultaneously to avoid interruptions to the mental health patient's train of thought. A different type of simultaneous interpreting takes place in international courts and tribunals (Stern 2012) where interpreters interpret bidirectionally for the question and answer sequences as well as for monologic segments using standard simultaneous interpreting equipment. This type of technology-assisted interpreting is not common in domestic courts and tribunals (Hale and Stern 2011), although it has been used in high profile trials as a one-off practice (Martin and Taibi 2012). A hybrid 
type of simultaneous interpreting is used in some domestic courts, in particular in the USA, where interpreters carry their own hand-held radio equipment in order to interpret what is normally interpreted in the whispering mode to the defendant. The use of this portable equipment allows the interpreter to sit anywhere in the courtroom rather than having to sit adjacent to the defendant, whispering in their ear (Stern, Ozolins, and Hale 2015).

The Australian Recommended National Standards for Working with Interpreters in Courts and Tribunals (RNS) (Judicial Council on Cultural Diversity 2017) recommended simultaneous interpreting with the use of adequate equipment for long trials. If these recommendations are implemented, simultaneous interpreting in courts in Australia may increase. There are noticeable benefits associated with the simultaneous mode: the interpreting does not add to the duration of the proceedings; the speech is not interrupted by the interpreting; and the speakers are less likely to get distracted by the interruptions and by the presence of the interpreter. The major disadvantage of consecutive interpreting is that it requires the interpreter to interrupt the speakers in order to interpret. These interruptions disrupt the flow of questioning and double the duration of the information exchange (Ewens et al. 2014; Hale et al. 2017). This process is also ill-suited to questions that yield a lengthy narrative response, or questioning techniques that rely on uninterrupted narrative responses (Powell et al. 2017). For the sake of accuracy, in consecutively interpreted interviews, interpreters need to interrupt the speaker who is delivering a narrative, so as to interpret the answer in manageable chunks, rather than wait for the end of the narrative in progress (Licoppe, Verdier, and Veyrier 2018). This means that turn-taking concerns and management issues may become relevant to the interpreter as the answer unfolds (Hale, GoodmanDelahunty, and Martschuk 2020), and that specialized interviewing techniques may be compromised (Goodman-Delahunty, Martschuk, Hale and Brandon, 2020; Powell et al. 2017). 
Simultaneous interpreting, by contrast, permits the exchange between interviewer and interviewee to proceed more smoothly and rapidly, without these interruptions. Previous research showed that the simultaneous interpreting mode is more conducive to interpreters achieving their aim of placing the non-mainstream language speaker in the same position as a mainstream language speaker, with minimal interference by the interpreter. An experimental study (Hale et al. 2017) compared the effect of consecutive and simultaneous interpreting on juror evaluations of witness credibility, yielding no significant differences between perceived credibility in the simultaneously interpreted and the monolingual control conditions, but significant differences between the consecutively interpreted and the monolingual control conditions. The same study showed that the consecutive condition interfered with the ability of the mock jurors to remember case facts accurately, as well as to concentrate.

Simultaneous interpreting in police settings has not been a common practice and has received very little research attention. A survey of 299 U.S. law enforcement practitioners revealed that $71 \%$ had conducted interpreted interviews in the consecutive mode, $12 \%$ in the simultaneous mode, $2 \%$ in both modes, and as many as $15 \%$ were unaware of the meaning of these terms (Shaffer and Evans 2018). In a field experiment on police interpreting, while the consecutive mode was the default mode used, some interpreters resorted to simultaneous whispering interpreting as an interactional management strategy when they encountered overlapping speech to maintain greater interpreting accuracy (Hale, Goodman-Delahunty, and Martschuk 2020).

This paper reports the results of a section of a larger experimental study about interpreting in police settings (Goodman-Delahunty et al. 2018), concentrating on the differences in interpreter performance according to interpreting mode. To our knowledge, this study is the first to explore the effects of simultaneous versus consecutive interpreting modes in the context of a police interview. 


\section{Cognitive differences between consecutive and simultaneous interpreting}

Among interpreting scholars, the widely-held belief is that simultaneous interpreting (SI) is cognitively more complex than consecutive interpreting (CI) (Gile 1995; Köpke and Nespoulos 2006; Meuleman and Van Besien 2009; Moser-Mercer 1997; Rinne et al. 2000; Yamada 2019). Interpreters themselves also perceive SI to be more stressful and more difficult (Korpal 2016). The reason for this view is that interpreters perform multiple complex tasks simultaneously: listening, understanding, memorising, converting and interpreting (Murphy and Myors 1999).

Gile advanced the interpreting Effort Models in the 1980s, which he revised and refined over the years $(2009,2018)$. The efforts are functions that compete for limited attentional resources or processing capacity. The models are based on the tightrope hypothesis that "Interpreters tend to work close to saturation" (Gile 2018, 10) especially in SI, in which all required conditions need to be met in order to perform at optimal levels. When one or more conditions fail to be met, "errors, omissions and/or infelicities" (EOIs) (Gile 2018, 10) can occur. EOIs can occur, not necessarily because of the interpreter's "insufficient knowledge of the working languages or the topics or insufficient technical skills but because attentional resources required to perform adequately were not available for a particular comprehension, memory storage or retrieval or production task" (Gile, 2018, 9).

The effort model for SI involves the Listening and Analysis Effort, the Short-term Memory Effort, the Speech Production Effort and the Coordination Effort. The CI Model involves Comprehension and Reformulation Efforts. However, although CI includes fewer steps, the CI model includes note taking and note reading, which are absent from the SI model. Whereas the efforts in SI compete with each other temporally in a concurrent way, in CI, the efforts compete with each other in a linear way, with intricate note taking creating the 
highest cognitive challenges during the comprehension phase, leading to potential errors and omissions, rather than infelicities. Notably, however, this type of CI is the long, classic interpreting mode, rather than the short consecutive mode used in dialogue interpreting.

Research on the cognitive load associated with interpreting has mostly concentrated on the work of simultaneous interpreters ( Barik 1973; Gerver 1969; Moser-Mercer 1997), whereas scholarly work on consecutive interpreting has concentrated mostly on note taking issues (Pöchhacker 2011a). This fact may indicate that assumptions about greater cognitive load for SI may not be empirically substantiated. Russell and Takeda (2015) contend that CI may be just as complex as SI. This opinion was empirically corroborated by a recent study of remote interpreting in a police interview via video link, which compared the visual attention of interpreters interpreting in the CI and SI modes, using eye-tracking technology. Doherty et al. (Doherty et al. forthcoming-a) found that simultaneous interpreters fixed their gaze on the speakers significantly more than did consecutive interpreters, whose visual attention was divided between their notes and the speakers, resulting in significantly more shifts of attention and arguably more cognitive load as a result of these shifts (see Henderson 2011). A significant correlation emerged between interpreter performance and interpreter gaze time: the longer the interpreters focused their visual attention on the speaker, the better their interpreting performance, and the more likely multisensory integration was to occur (see Doherty et al. forthcoming-a). A further finding was that the simultaneous interpreting mode was more conducive to longer fixated gaze on the speakers, thereby producing more accurate results, whereas the consecutive mode forced interpreters to divide their gaze between their notes and the speakers, producing less accurate interpreting outcomes (Doherty et al. forthcoming-a). In a separate study (Doherty et al. forthcoming-b), the authors used two physiological measures of higher cognitive load, pupillometry and blink rate, to compare modes. The percentage change in median pupil size and blink rate relative to individual 
baselines were elevated in consecutive interpreting. These results were consistent in remote interpreting via video link and via audio.

These recent findings add to the evidence-based discussion on interpreting mode and performance. Moreover, there is also an element of habituation to a particular mode in which the complex language process becomes more efficient with practice. Consequently, the cognitive load in the mode with which an interpreter is most accustomed may reduce while that of the mode with which an interpreter is not accustomed may increase (see Kruger and Doherty 2016). Performance accuracy, of course, would need to be taken into account as higher cognitive load does not always correlate with better performance and vice versa.

\section{Differences in accuracy between consecutive and simultaneous interpreting}

Regardless of which mode requires more cognitive effort, an important aspect that has not yet been determined is whether the same level of interpreting accuracy can be achieved irrespective of mode. The findings of the few studies on the impact of mode on interpreting accuracy are contradictory (Berk-Seligson 1999; Gile 2001; Hale et al. 2017; Russell 2002), with some suggesting better outcomes for CI and some for SI. For example, in a US study of the interpretation of 945 authentic recorded coercive courtroom questions, CI elicited more accurate interpretations into Spanish, with its accuracy level reaching 71\% compared to only 33\% for SI (Berk-Seligson 1999). A similar result was observed in an experimental study of video recorded mock trials where certified American Sign Language interpreters used the two different modes. Despite the fact that the simultaneous mode is the default mode for signed language interpreting, the interpreters in this study consistently performed better in the consecutive mode (Russell 2003). By contrast, in two other studies, SI produced the most accurate results. In a healthcare setting, an experimental study showed that SI on the telephone produced more accurate renditions than CI either by telephone or face-to-face (Gany et al. 2007). In a study of 20 professional conference interpreters, in which half 
interpreted in the simultaneous mode and half in the consecutive mode, a panel of judges assessed the performance of the simultaneous interpreters to be more accurate to the speaker's delivery and style, whereas the consecutive interpreters tended to omit many of the speakers' paralinguistic features and to offer a more concise version of the content, which is to be expected in the classic consecutive interpreting mode, for which interpreters must rely on intricate notes and their short-term memory to deliver the interpretation (Gile 2001).

Interpreting training programs have traditionally taught consecutive interpreting before simultaneous interpreting, based on the assumption that simultaneous interpreters first need to master the consecutive interpreting mode before moving on to the more difficult mode of simultaneous interpreting (Yamada 2019). Arguably, the order in which these modes are taught in the curriculum may have something to do with the historical and chronological development of the modes, with CI being the first professional mode, followed by SI, which was only introduced with the advent of equipment. The professional interpreters of the time needed to be trained to use the new mode, and training proved to be successful in achieving better outcomes (Seeber 2015). Yamada (2019) argues that novice interpreters may not need to be trained in CI before being introduced to SI. In a study of 20 beginner English to Japanese interpreting students, in a one-semester course in which all the modes were taught concurrently, at the end of the semester, the students were able to interpret simultaneously relatively well, without substantial prior consecutive interpreting training (Yamada 2019). This finding may indicate that both modes are equally complex for different reasons.

\section{The Present Study}

In light of the inconclusive results of previous research on the effect of interpreting mode on interpreter performance, and in order to fill the knowledge gap in police settings, our study aimed to answer the following four questions: 
1. Does the consecutive interpreting mode lead to more accurate interpreting than the simultaneous mode?

2. Do language combinations moderate the performance of similarly qualified interpreters?

3. Does experience in simultaneous interpreting in legal settings increase interpreting accuracy in SI?

4. Which mode of interpreting do interpreters perceive to require more mental effort?

Based on the foregoing literature review, our hypothesis for the first question was that accuracy in the consecutive interpreting mode would exceed that in the simultaneous mode. This is because the consecutive mode is the default mode for police interpreting, is the predominant mode taught in training programs and is perceived by theorists and practising interpreters to be less complex than simultaneous interpreting.

Our hypothesis for the second question was that language would not make a meaningful difference in the performance of interpreters by interpreting mode (when their training, qualification and experience were controlled), but that differences between languages by mode would be negligible.

Our hypothesis for the third question was that practitioners with simultaneous interpreting experience in the field in legal settings would perform better than interpreters without this field experience in SI (Wong 2020).

Our hypothesis for the fourth question, was that interpreters would perceive simultaneous interpreting as the more cognitively challenging mode. 


\section{Method}

Research Design

The experimental study employed a 3 x 2 mixed design. Language had three levels (Spanish vs. Arabic vs. Mandarin) and was varied between-participants. Interpreting Mode had two levels (simultaneous vs. consecutive) and was manipulated within participants.

\section{Participants}

Participants were recruited via a call for participants in the relevant languages sent to AUSIT $^{1}$ members and all NAATI ${ }^{2}$ accredited interpreters. The educational institutions that teach Interpreting also distributed the call among their final year students. Although there is no way of knowing exactly how many interpreters received the call for participants, the current NAATI directory lists 268 certified Mandarin interpreters, 152 certified Arabic interpreters and 46 certified Spanish interpreters in the state of New South Wales. However, not all of the interpreters can be assumed to be graduates of formal tertiary interpreting programs.

A total of 70 qualified interpreters participated in the study (32 Mandarin-, 25 Spanish-, and 13 Arabic-speaking), of whom $80 \%$ were women and $20 \%$ were men. Their mean age was 41.39 years $(S D=14.20)$, with an age range between 21 and 73 years. Mandarinspeaking interpreters were on average younger $(M=35.60, S D=11.05)$ than Arabic- $(M=$ 43.50, $S D=11.12, p=.035)$ and Spanish-speaking interpreters $(M=46.91, S D=15.95, p=$ $.001 ; F(2,102)=7.71, p=.001)$. Forty percent of the participants had completed a Master's degree or a Graduate Diploma in interpreting, and a further $15.7 \%$ had completed a Bachelor's degree in interpreting; $24.3 \%$ reported completion of a Vocational Education and Training (TAFE) diploma or advanced diploma (12.6\% and 16.5\%, respectively). Seventeen

\footnotetext{
${ }^{1}$ AUSIT is the Australian Institute for Interpreters and Translators, the national professional association. ${ }^{2}$ NAATI is the National Accreditation Authority for Translators and Interpreters. At the time of the study, interpreters were accredited by NAATI. The system changed in 2018, after which interpreters become certified.
} 
percent had not received any formal interpreting educational training but had passed the NAATI professional accreditation test, and a further 3\% had attended short professional development or university non-award courses. Sixteen percent were interpreting students, of whom most (11.4\%) had finished their coursework and were graduating in the same year. Interpreters' practical experience was on average 7.81 years $(S D=8.70)$, ranging between zero for the graduating students and 33 years.

The majority of the interpreters had received training in both modes. One hundred percent of the Arabic, $96 \%$ of the Spanish and $85 \%$ of the Mandarin interpreters had received formal training in CI. Simultaneous interpreting training was not as common, although still very prevalent for Spanish (83\%) and less frequent for Arabic and Mandarin interpreters (69\%). The Spanish interpreters were the most experienced in SI (64.0\%), followed by the Arabic (53.8\%) and the Mandarin (37.5\%) interpreters. Close to half of Spanish and Arabic interpreters (48.0\% and $46.2 \%$, respectively) and $31.3 \%$ of Mandarin interpreters reported having used SI in legal settings, such as in court, police interviews, and/or tribunals. Table 1 provides an overview of the interpreters' professional background by language group.

Table 1

Demographic Characteristics of Participant Interpreters (\%)

\begin{tabular}{ccc}
\hline Spanish & Mandarin & Arabic \\
$(n=25)$ & $(n=32)$ & $(n=13)$ \\
\hline
\end{tabular}

Formal interpreting training*

None

20.0

15.6

17.1

Short courses/non-award courses

TAFE diploma / advanced

diploma

20.0

21.9

38.5

University degree

56.0

62.5

38.5

Specific interpreting courses

Consecutive

95.7

100.0

84.6

Simultaneous

82.6

69.2

69.2

NAATI accreditation *

None or not specified

20.0

Paraprofessional

20.0

21.9

46.2

Professional

60.0

78.1 
Interpreting experience

$\begin{array}{lrrr}\text { Simultaneous interpreting* } & 64.0 & 37.5 & 53.8 \\ M \text { [years] } & 10.72 & 6.44 & 5.62 \\ S D \text { [years] } & 11.40 & 6.75 & 5.50 \\ \text { Range [years] } & 0-33 & 0-30 & 0-18\end{array}$

Gender

\begin{tabular}{cccc} 
Female & 76.0 & 87.5 & 69.2 \\
\hline Note. ${ }^{*}$ significant differences between language groups $(p<.05) ; M=$ mean; $S D=$ standard deviation.
\end{tabular}

\section{Interview Simulation Materials}

Interview script. A script depicting an investigative interview between an English-speaking police interviewer and a non-English-speaking suspect who was charged with financing a terrorist organisation was developed in consultation with police practitioners and experts in interpreting to ensure that the content tested a series of realistic interpreting challenges. The interview was approximately 2,000 words in length in English. The script was divided into two halves. Each half contained equivalent interpreting challenges, e.g., formal legal language used by the detective, colloquial Australian expressions, such as the professed occupation of the suspect ("brickie" i.e., bricklayer), and profane language in the responses of the suspect (see Hale et al. 2020 for the results of the study on the interpretation of profane language). The master script was written in English and translated into three languages by NAATI accredited, trained translators. Professional actors performed the roles of police interviewer and Arabic-, Mandarin- and Spanish-speaking suspects. The actors were instructed to follow the script but to adapt it in response to the interpreters' interpretation. Post-interview questionnaire. Participants responded to brief questions about their professional and educational background as interpreters, and their perceived mental effort in the interpreting task. The participants rated their mental effort on a scale $(1=$ very low, $7=$ very high), when interpreting consecutively, simultaneously, from English, into English, and while taking notes (if they did). Further, they rated the difficulty of the interpreting task in 
general $(1=$ not at all, $7=$ very $)$, and their impressions of the interactions between the parties and the interviews. Here we report only on the interpreters' perceived mental effort in performing the simultaneous and the consecutive interpreting tasks.

\section{Procedures}

The research was approved by the Federal Bureau of Investigation Institutional Review Board (378-16), the Charles Sturt University Human Research Ethics Committee (H16164), and the University of New South Wales Human Research Ethics Committee (H16164). Before commencing the simulated interview, interpreters were instructed that they would be interpreting between an English-speaking police interviewer and an Arabic-, Mandarin-, or Spanish-speaking suspect who did not speak English. Half of the participants were instructed to start interpreting using the simultaneous mode; the other half were instructed to start interpreting using the consecutive mode. The order in which the interpreting mode was administered was counter-balanced across participants to control for any potential order effects caused by the experimental condition. After the interview concluded, the participants completed a brief written questionnaire. On completion of the questionnaire, interpreters were debriefed and received a $\$ 100$ gift voucher to compensate them for their time.

The interviews were video- and audio-recorded and transcribed for later analysis. The transcriptions were conducted by professional transcriptionists according to detailed guidelines prepared by the researchers. The transcriptions included the propositional content as well as other relevant features such as hesitations, corrections, backtrackings, overlapping speech, side comments, and marked for mode of interpreting (consecutive or simultaneous).

\section{Data Analysis}

Assessment of interpreting performance. Each transcript was coded by trained coders in each language. 
Each transcript was assessed for interpreting performance using a set of weighted criteria routinely applied in oral interpreting examinations to assess legal interpreting students by one of the authors (see Table 2). The same assessment criteria used in a previous study (Hale, Goodman-Delahunty, and Martschuk 2020) reliably distinguished trained interpreters from ad hoc bilinguals. 
Table 2

Coded Variables and Interpreting Performance Assessment

\begin{tabular}{|c|c|c|c|}
\hline $\begin{array}{l}\text { Element of } \\
\text { competence / coded } \\
\text { variable }\end{array}$ & Criterion descriptors & Mark & Weight \\
\hline $\begin{array}{l}\text { Accuracy of } \\
\text { propositional content }\end{array}$ & $\begin{array}{l}\text { The interpreter maintains the content of the } \\
\text { utterance, 'what' the speaker said. }\end{array}$ & 10 & $30 \%$ \\
\hline Accuracy of style & $\begin{array}{l}\text { The interpreter maintains stylistic features, the } \\
\text { 'how' of the utterance. This includes pragmatic } \\
\text { force (tone, intonation, stress, hesitations, fillers, } \\
\text { hedges, repetitions, etc.) and maintenance of } \\
\text { register (formal/informal, technical/colloquial). }\end{array}$ & 10 & $15 \%$ \\
\hline $\begin{array}{l}\text { Maintenance of verbal } \\
\text { rapport markers }\end{array}$ & $\begin{array}{l}\text { The interpreter maintains the rapport features of } \\
\text { the original. These include use of first name, } \\
\text { acknowledgement markers such as 'OK' at the } \\
\text { start of a response, politeness markers such as } \\
\text { 'please' and 'thank you', expressions of solidarity } \\
\text { and comfort. }\end{array}$ & 10 & $15 \%$ \\
\hline $\begin{array}{l}\text { Use of correct } \\
\text { interpreting protocols }\end{array}$ & $\begin{array}{l}\text { The use of the direct approach }\left(1^{\text {st }} \& 2^{\text {nd }}\right. \\
\text { grammatical persons), interpreting everything that } \\
\text { is said regardless of what it is, seeking repetitions } \\
\text { when needed in the right way, transparency } \\
\text { (keeping everyone informed if a repetition or } \\
\text { clarification is required). }\end{array}$ & 10 & $10 \%$ \\
\hline $\begin{array}{l}\text { Legal discourse and } \\
\text { terminology }\end{array}$ & $\begin{array}{l}\text { Maintaining institutional phrases and grammatical } \\
\text { structures, correct use of strategic question types, } \\
\text { legal formulas and correct legal terminology. }\end{array}$ & 10 & $10 \%$ \\
\hline $\begin{array}{l}\text { Management and } \\
\text { coordination skills }\end{array}$ & $\begin{array}{l}\text { This includes setting the contract by establishing } \\
\text { the interpreter's role and modus operandi, knowing } \\
\text { when to interpret and how to manage the } \\
\text { interaction. }\end{array}$ & 10 & $10 \%$ \\
\hline Language competence & $\begin{array}{l}\text { Grammatical correctness, correct pronunciation, } \\
\text { fluency in both languages }\end{array}$ & 10 & $10 \%$ \\
\hline Total mark & & 70 & $100 \%$ \\
\hline
\end{tabular}

The assessment distinguished seven weighted criteria, as shown in Table 2, summing to $100 \%$. To compare the level of accuracy by mode each half of the transcript was assessed separately out of 100. Each transcription was scored by two trained raters using detailed descriptors for each criterion (Table 2). The average inter-rater reliability score was good (Krippendorff $\alpha=.85$ ). When the agreement between two raters was low (i.e., below Krippendorff $\alpha=.7$ ), a third independent rater assessed the transcripts. The final assessment 
score was the average value between two coders. If a third coder was involved, the average between the results from the two raters who achieved the highest interrater reliability scores was used.

\section{Results}

Performance by Interpreting Mode Using the Seven Assessment Criteria

A mixed between-within participants ANOVA with interpreting mode as a withinparticipant variable and language as a between-participant variable revealed a main effect for interpreting mode (Wilks' $\left.\Lambda=.905, F[1,67]=7.03, p=.010, \eta_{p}^{2}=.10\right)$, showing better interpreting performance when the simultaneous mode was used $(M=69.15, S D=10.71$, $95 \% \mathrm{CI}[66.44,71.87])$ than the consecutive mode $(M=67.03, S D=11.08,95 \% \mathrm{CI}[64.21$, 69.86]). This effect held across the three languages for the overall performance scores out of 100 (Wilks' $\left.\Lambda=.965, F[2,67]=1.22, p=.302, \eta_{p}^{2}=.04\right)$. The minimum-effect hypothesis that language accounts for less than $1 \%$ of the variance in outcomes was confirmed: The approximate $F$ value $F_{\text {approx }}(2,67)=3.37$ was below the critical $F$ value $F_{\text {critical }}(2,70)=4.14$ (Murphy and Myors 1999).

When the different criteria to measure interpreting performance were considered separately, the analyses revealed statistically significant differences between the simultaneous and the consecutive modes for three of the criteria listed in Table 2: interpreting style (Wilks' $\left.\Lambda=.925, F[1,67]=5.46, p=.022, \eta_{p}^{2}=.08\right)$; maintenance of verbal rapport markers (Wilks' $\left.\Lambda=.930 F[1,67]=5.04, p=.028, \eta p^{2}=.07\right)$, and use of correct interpreting protocol, Wilks' $\left.\Lambda=.906, F[1,67]=6.94, p=.010, \eta p^{2}=.09\right)$, with SI producing statistically significantly better outcomes. The interactions between interpreting mode and language were not significant on any of the dependent measures. Figure 1 shows the weighted scores for each criterion combined for all interpreters, by interpreting mode. 


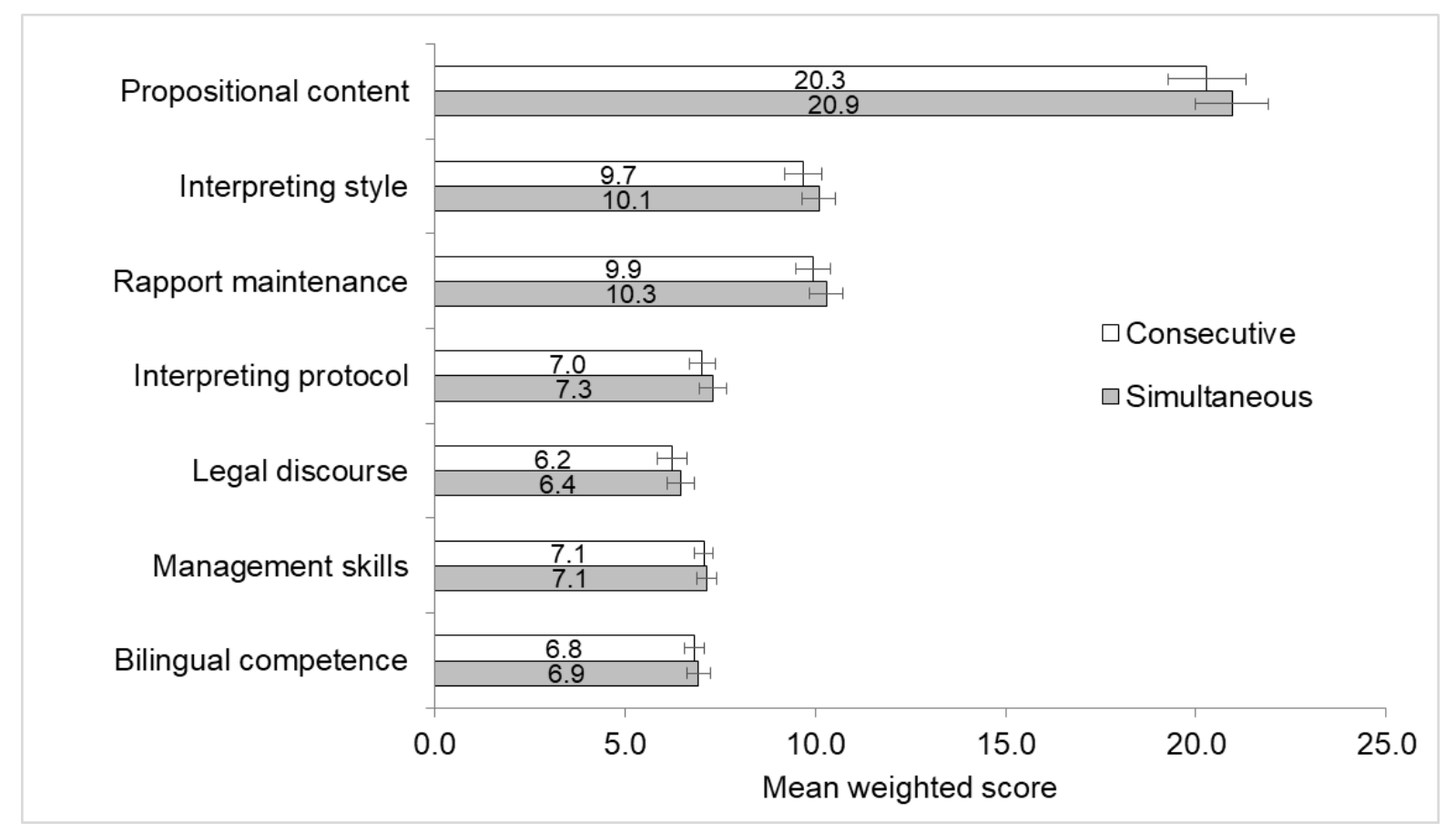

Figure 1. Mean weighted scores by interpreting mode (error bars are $95 \%$ confidence intervals).

Figure 2 shows the mean difference between the accuracy scores for SI and CI for all interpreters on each assessment criterion. A positive value means that interpreters performed better in SI than in CI. A value of 0 indicates no differences between the modes, while a negative value indicates better performance in CI than in SI. As can be seen in Figure 2, all values on all assessment criteria are positive, showing that performance scores in SI exceeded those in CI on all seven measures. 


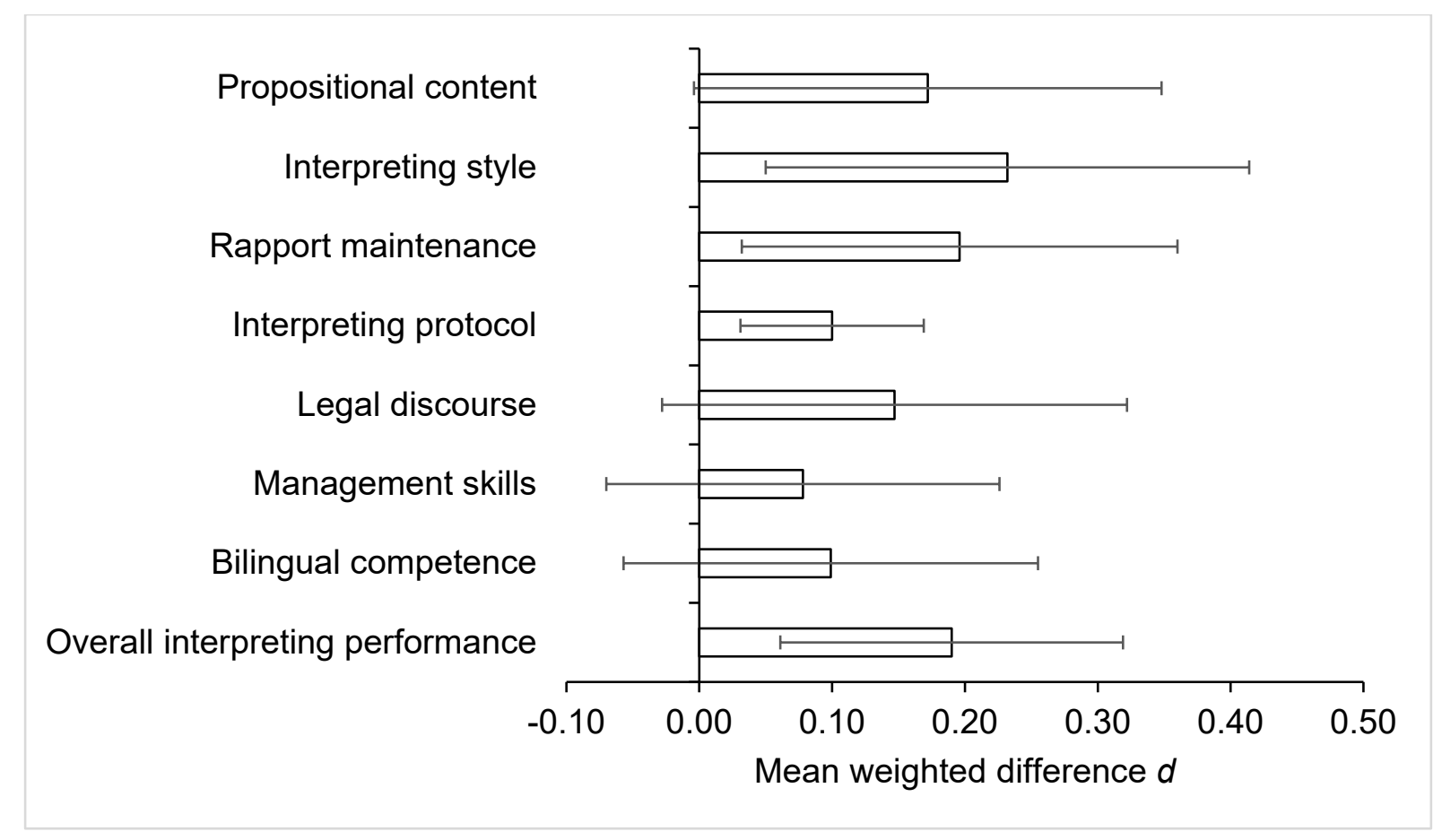

Figure 2. Mean weighted difference between the simultaneous and the consecutive interpreting modes (error bars are 95\% confidence intervals).

In response to research question 1, whether CI leads to more accurate interpreting than SI, our results showed that interpreters performed better in the simultaneous mode, contrary to hypothesis 1 . In response to research question 2 , whether language combinations moderate the performance of similarly qualified interpreters, our results of negligible differences across languages confirmed hypothesis 2 . 
While the analyses above contrasted SI and CI, in this section we further explored performance of interpreters in the simultaneous interpreting mode as a function of their experience. Therefore, interpreters were divided in two groups: (a) interpreters with no SI experience in legal settings; and (b) interpreters with SI experience in legal settings, such as police interviews, criminal trials, or tribunals.

Analysis of covariance on the overall interpreting performance score assessed the difference between interpreters experienced in SI in legal settings and those who lacked SI experience in legal settings, while controlling for years of interpreting experience. Interpreters experienced in SI in legal settings performed significantly better at SI $(M=$ $72.44, S D=10.85,95 \%$ CI $[68.38,76.49])$ than those who lacked SI experience in legal settings $\left(M=65.94, S D=9.86,95 \%\right.$ CI $\left.[62.66,69.22], F[1,67]=5.86, p=.018, \eta_{p}^{2}=.08\right)$.

A one-way multivariate analysis of covariance assessed interpreting performance on the seven different assessment criteria as a function of SI experience in legal settings, while controlling for years of interpreting experience. Results revealed statistically significant differences between interpreters with and without SI experience in legal settings (Wilks' $\Lambda=$ $\left..776, F[7,61]=2.52, p=.024, \eta p^{2}=.22\right)$. When the assessment criteria were considered separately, statistically significant differences emerged for three performance criteria: interpreting style, $F(1,67)=5.60, p=.021, \eta_{p}^{2}=08$; accurate interpreting of verbal rapport, $F(1,67)=9.43, p=.003, \eta_{p}{ }^{2}=.12$; and the use of correct interpreting protocols, $F(1,67)=$ 9.27, $p=.003, \eta_{p}^{2}=.12$. As hypothesised (H3), interpreters with SI experience in legal settings were more likely to accurately render the style of the source utterance $(M=10.63$, $S D=1.54,95 \%$ CI $[10.00,11.26])$, accurately interpret verbal rapport features $(M=10.92$, $S D=1.58,95 \% \mathrm{CI}[10.28,11.56])$, and use the correct interpreting protocol $(M=7.80, S D=$ $1.28,95 \% \mathrm{CI}[7.27,8.34])$, than interpreters without this experience (Interpreting style: $M=$ 
9.64, $S D=1.68,95 \%$ CI $[9.13,10.15]$; verbal rapport maintenance: $M=9.62, S D=1.73$, 95\% CI [9.10, 10.13]; correct interpreting protocol: $M=6.72, S D=1.54,95 \%$ CI $[6.29$, 7.16]). The differences between interpreters with and without SI experience in legal settings were not statistically significant for the remaining assessment criteria. Figure 3 shows the weighted scores for each criterion combined for all interpreters, by SI experience in legal settings.

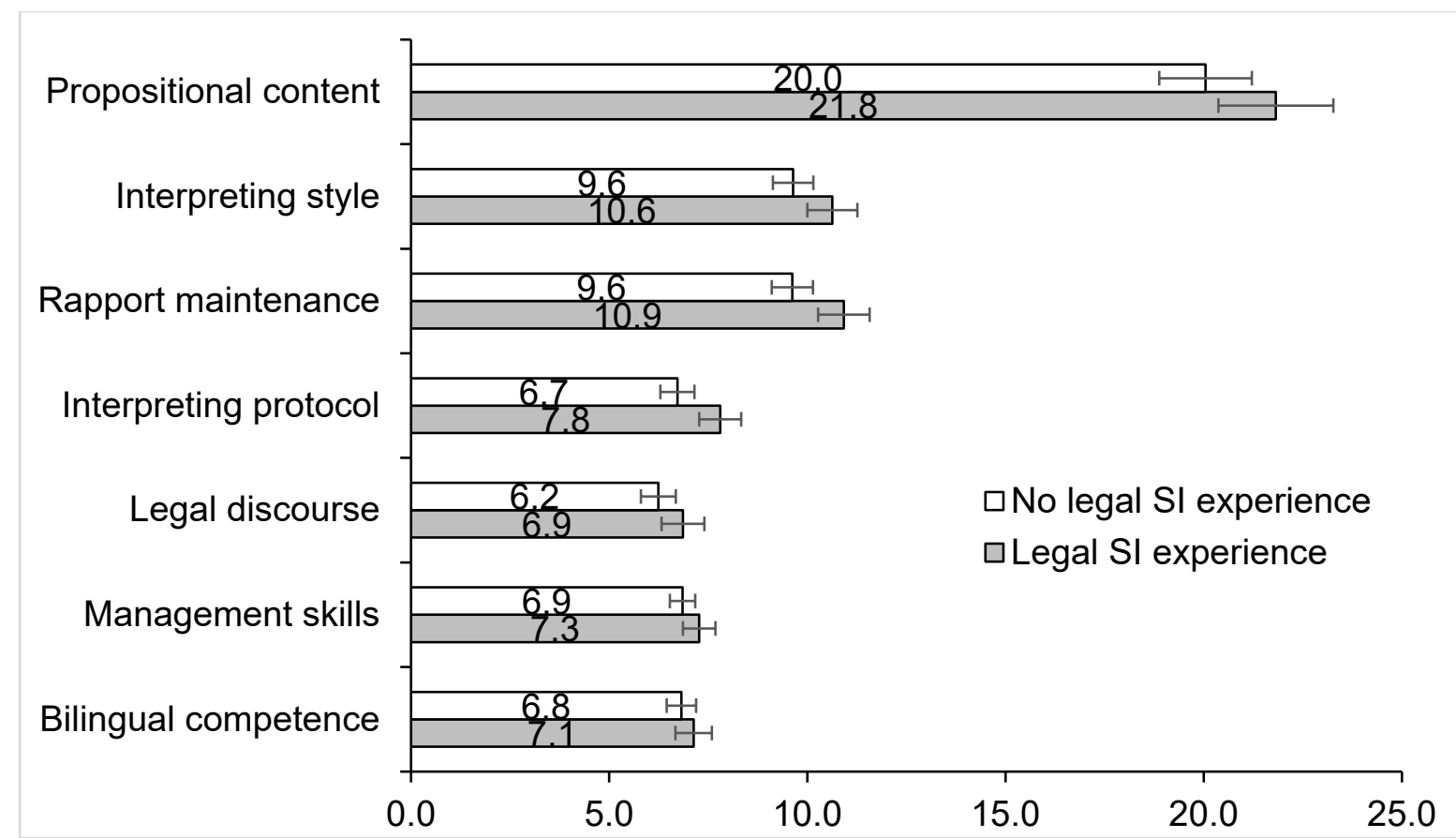

Figure 3. Mean weighted scores by SI experience in legal settings (error bars are $95 \%$ confidence intervals).

In response to research question 3 , whether experience in simultaneous interpreting in legal settings increases interpreting accuracy, our results showed that experience made a positive difference in performance, confirming hypothesis 3 . 


\section{Post-experiment Interpreter Survey}

In rating their perceived mental effort interpreters reported greater mental effort in SI $(M=4.74, S D=1.71)$ than in $\mathrm{CI}(M=3.83, S D=1.93)$. The perceived mental effort exerted in the two modes was not significantly correlated $(r=.20, p=.098)$. Interestingly, greater perceived mental effort in SI was not matched to interpreting performance measures, which did not show an association $(r(69)=-.12, p=.345)$. Similarly, the lesser perceived mental effort in CI was not significantly associated with overall interpreting performance $(r(70)=$ $.02, p=.902)$. These results indicated that the interpreters' subjective perceptions of effort exerted were unrelated to their interpreting scores as assessed by independent raters. This result is unsurprising given the cognitive bias associated with complex judgement tasks when one's cognitive capacity is already exhausted by the task itself, thereby limiting the ability and accuracy of one's appraisal of one's own performance (Razon, Hutchinson, and Tenenbaum 2012). In response to research question 4, which interpreting mode interpreters perceive to produce the highest level of mental effort, the results confirmed our hypothesis that interpreters perceived SI as more cognitively demanding, although their performance was unrelated to their perceptions.

\section{Discussion}

This study investigated the impact of mode (consecutive vs simultaneous), language pair (English and Arabic, Mandarin or Spanish) and interpreting experience on interpreting performance in a simulated interpreted police interview. It also explored interpreters' selfperceptions of mental effort exerted according to mode. To avoid a possible artefact of sample bias, the study compared the consecutive and simultaneous interpreting performance scores of each individual interpreter (within-participant design). The results showed that overall, the interpreters in our sample performed better in the simultaneous mode on three of 
the seven assessed criteria, with those experienced in legal settings achieving the best performance assessments. The results emerged irrespective of the language combination, although the magnitude of the observed differences using this conservative experimental procedure was modest, i.e., the effect sizes were small. This result can be attributed to the within-participant design, which compares the performance of the same interpreter in different modes. All interpreters were trained on both CI and SI modes. Thus, the same interpreter is likely to exhibit a similar level of core competencies and skills, regardless of mode. Arguably, although the observed effect sizes are small, our study used a very stringent test to detect the difference that mode makes in an individual interpreter's performance.

The statistically significant differences found within-participant performance related to three assessment criteria: accurate interpreting of style, of rapport features, and use of the correct interpreting protocol. The fact that these three features are essential in contemporary police interviewing techniques make the results particularly important. The finding on style replicated that in the performance of conference interpreters, in which the judges assessed SI as more accurately rendering the style of the source speaker, compared to CI, which tended to summarise and omit the source speaker's paralinguistic or stylistic features (Gile, 2001).

Notably, through their self-assessment on the Likert-type scale, the interpreters in our sample expressed the long-held view that SI requires a higher cognitive load than CI (Köpke and Signorelli 2012). Our findings, however, indicated that this viewpoint may reflect a common misconception, as corroborated by recent and consistent empirical findings (see also Doherty et al. forthcoming-a).

This study's main finding that SI overall leads to better or equally good interpreting performance in police settings is heartening in light of other widely-acknowledged advantages of this mode, such as the shorter duration of the interaction time, and fewer interruptions of the speakers. By systematically comparing the two major interpreting modes, 
the study contributed to the resolution of prior conflicting outcomes on advantages of one interpreting mode over another (Berk-Seligson 1999; Hale and Stern 2011; Russell 2002), in the absence of any controlled comparison of the two interpreting modes (Hornberger et al. 1996). However, it is important to stress that our sample comprised trained interpreters and that specialised experience in legal settings led to better performance in SI.

\section{Strengths and Limitations}

A particular strength of this study is the multi-pronged analysis undertaken to assess interpreting performance, which enabled a robust analysis in a realistic simulated interview. In comparison to other simulations, this interview was lengthier than those used in many prior studies, thus allowing a more robust assessment of performance. The experimental context was high in ecological validity compared to other simulated law enforcement interviews. It included a series of question-answer exchanges between the speakers and was modelled on an authentic transcript of an investigative interview conducted with a nonEnglish-language suspect in a real Australian criminal case.

The study also has limitations. One limitation was the difficulty in ensuring that exactly the same script was followed. Using trained actors minimised the differences. However, to ensure ecological validity, the actors were required to adapt the script according to the interpreters' renditions. This inevitably led to slight differences across the participants.

Although our study is larger than most others to date, the sample is still relatively small, assessing only three different language combinations from only one geographical location, thus limiting the generalizability of the results. Further large studies using different language combinations should be conducted.

Other limitations of the study that must be considered are features of the research design and the measures of interpreting accuracy. As was noted above, the small effect sizes obtained in comparing SI and CI performance accuracy were likely attributable to the within- 
interpreter comparisons applied in this research design. Greater differences may emerge when comparing two different groups of interpreters performing either in the consecutive or the simultaneous mode and averaging the results, as there would likely be more variability in group performance ratings on average than differences between one individual's performance in each of the two modes. However, a difficulty in interpreting the results of between group differences is the extent to which they are attributable to differences in the interpreters' base interpreting skills, rather than to the mode. An advantage of our within-participant design is that it controlled for base interpreting skill differences.

The small effect sizes observed may also be attributable to the nature of our interpreting performance measures. Although seven discrete criteria of interpreting performance were assessed, the rating scheme applied to each of those criteria was somewhat impressionistic. These dependent measures may have been less sensitive to fine distinctions in interpreting skills by the same interpreter in different modes than they would be to differences in modes between different interpreters, using a between-participants design. Unless an individual interpreter exhibited considerable differences in skill between modes, we would not observe great differences, yielding larger effect sizes using these accuracy measures.

A further limitation of our study is the single measure used to assess perceived mental effort. The focus of this paper was not interpreters' cognitive effort according to mode. We simply reported the interpreters' post-experiment perceptions of the mental effort exerted to ascertain whether their perception matched their performance. More in-depth analyses of cognitive load and mental effort have been undertaken (Doherty et al., forthcoming - a, b).

\section{Conclusions and Recommendations}

This study provided further insight into the debate about the difference that mode of interpreting can make on interpreter performance. The first central finding of this study was 
that SI produced better performance than CI in a number of ways, specifically accurate interpreting of style and rapport features, and use of correct interpreting protocols, which are especially critical in investigative interviews. The second central finding was that performance in different modes was unaffected by language combination, as revealed by our comparison of interpreting performance in three major global languages. Our study also confirmed that specialised experience in legal settings produced better performance in SI. Finally, the interpreters who participated in our study held the well documented perception that SI is more cognitively challenging than CI, even though they were assessed to have performed better in SI than in CI. These findings are important considering the many added benefits of SI, as expressed in our introduction. However, it is important to stress that in order to ensure high levels of performance, competent interpreters who are trained in SI should be employed, giving preference to those experienced in legal interpreting. The following suggestions flow from these findings:

1. Interpreting training courses should prepare interpreters to work in the simultaneous mode in legal contexts, and not only in the consecutive mode; and

2. Simultaneous interpreting equipment should be available in legal settings to facilitate the simultaneous mode and the independent recording of the different inputs.

Acknowledgements

This research was funded by the Federal Bureau of Investigation High-Value Detainee Interrogation Group Intelligence Interviewing and Interrogation Research contract DJF-161200-V-0003904 awarded to Charles Sturt University and the University of New South Wales. Statements of fact, opinion and analysis in the paper are those of the authors and do not reflect the official policy or position of the FBI or the U.S. Government. 
We gratefully acknowledge the work of our research officer, Dr Julie Lim, our research assistants, and the participating interpreters.

\section{References}

Baigorri-Jalon, J. 2011. "Back to the future." 15th DG Interpretation Universities conference, Interpreting in a globalized world, Brussels (17-18 Mar).

Barik, Henri C. 1973. "Simultaneous interpretation: Temporal and quantitative data." Language and speech 16(3): 237-270.

Berk-Seligson, Susan. 1999. "The impact of court interpreting on the coerciveness of leading questions." Forensic Linguistics 6(1): 30-56.

Doherty, Stephen M., Natalie Martschuk, Jane Goodman-Delahunty, and Sandra Hale. forthcoming-a. An eye-movement analysis of visual attention and interpreting performance during consecutive and simultaneous interpreting modes in a remotely interpreted investigative interview.

---. forthcoming-b. A pupillometric and blink rate analysis of cognitive load and interpreting performance during consecutive and simultaneous interpreting modes in a remoteinterpreted investigative interview.

Ewens, Sarah, Aldert Vrij, Sharon Leal, Samantha Mann, Eunkyung Jo, and Ronald P. Fisher. 2014. "The effect of interpreters on eliciting information, cues to deceit and rapport." Legal and Criminological Psychology: 1-19. https://doi.org/10.1111/lcrp.12067

Gany, Francesca, Luciano Kapelusznik, Kavitha Prakash, Javier Gonzalez, Lurmag Y. Orta, Chi-Hong Tseng, and Jyotsna Changrani. 2007. "The Impact of Medical Interpretation Method on Time and Errors." Journal of General Internal Medicine 22(2): 319-323. http://dx.doi.org/10.1007/s11606-007-0361-7

Gerver, David. 1969. "The effects of source language presentation rate on the performance of simultaneous conference interpreters." Proceedings of the 2nd Louisville Conference on rate and/or frequency controlled speech.

Gile, Daniel. 1995. Basic concepts and models for interpreter and translator training. Vol. 8Benjamins translation library. . Amsterdam: John Benjamins Publishing Company.

---. 2001. "Consecutive vs. Simultaneous: which is more accurate?" The Journal of the Japan Association for Interpretation Studies (1): 8-20.

---. 2009. Basic concepts and models for interpreter and translator training. Rev. ed. Vol. 8Benjamins translation library. Amsterdam: John Benjamins Publishing Company.

---. 2018. "The effort models and gravitational model: Clarifications and update [PowerPoint]." http://www.cirinandgile.com/powerpoint/The-Effort-Models-andGravitational-Model-Clarifications-and-update.pdf

Goodman-Delahunty, Jane, Natalie Martschuk, Sandra Hale, and Susan E. Brandon, 2020. "Interpreted police interviews: A review of contemporary research". In Advances in psychology and law (Vol. 5) edited by Monica Miller and Brian H. Bornstein. Springer.

Goodman-Delahunty, Jane, Natalie Martschuk, Sandra Hale, Stephen M. Doherty, and Mustapha Taibi. 2018. "Interpreter presence, mode and language in investigative interviews." Research report submitted to the High-Value Detainee Interrogation Group (HIG), USA. Charles Sturt University, Manly. 
Hale, Sandra. 2007. "The challenges of court interpreting: intricacies, responsibilities and ramifications." Alternative Law Journal 32(4): 198-202

Hale, Sandra, Jane Goodman-Delahunty, and Natalie Martschuk. 2020. "Interactional management in a simulated police interview: Interpreters' strategies." In The discourse of police interviews, edited by Marianne Mason and Frances Rock. Chicago: The University of Chicago Press.

Hale, Sandra, Natalie Martschuk, Jane Goodman-Delahunty, Mustapha Taibi, and Han Xu. 2020. "Interpreting profanity in police interviews." Multilingua 39(4): 369-393. https://doi.org/10.1515/multi-2019-0065

Hale, Sandra, Natalie Martschuk, Uldis Ozolins, and Ludmila Stern. 2017. "The effect of interpreting modes on witness credibility assessments." Interpreting: International Journal of Research and Practice in Interpreting 19(1): 69-96.

Hale, Sandra, and Ludmila Stern. 2011. "Interpreter quality and working conditions: Comparing Australian and international courts of justice." Judicial Officers Bulletin 23(9): 75-81.

Henderson, John M. 2011. "Eye movements and scene perception." In Oxford Handbook of Eye Movements edited by S. Liversedge, I. D. Gilchrist and S. Everling, 593-606. Oxford: Oxford University Press.

Hornberger, John C., Count D. Gibson, William Wood, Christian Dequeldre, Irene Corso, Barbara Palla, and Daniel A. Bloch. 1996. "Eliminating Language Barriers for NonEnglish-Speaking Patients." Medical Care 34(8): 845-856.

Jacobsen, Bente. 2012. "The significance of interpreting modes for question-answer dialogues in court interpreting." Interpreting 14(2): 217-241.

Judicial Council on Cultural Diversity. 2017. Recommended National Standards for Working with Interpreters in Courts and Tribunals Judicial Council on Cultural Diversity (Canberra). http://jccd.org.au/wp-content/uploads/2018/02/JCCD-InterpreterStandards.pdf

Köpke, Barbara, and Jean-Luc Nespoulos. 2006. "Working memory performance in expert and novice interpreters." Interpreting 8(1): 1-23.

Köpke, Barbara, and Teresa M. Signorelli. 2012. "Methodological aspects of working memory assessment in simultaneous interpreters." International Journal of Bilingualism 16(2): 183-197.

Korpal, Paweł. 2016. "Interpreting as a stressful activity: Physiological measures of stress in simultaneous interpreting." Poznan Studies in Contemporary Linguistics 52(2): 297316.

Kruger, Jan-Louis, and Stephen Doherty. 2016. "Measuring cognitive load in the presence of educational video: Towards a multimodal methodology." Australasian Journal of Educational Technology 32(6): 19-31.

Licoppe, Christian, Maud Verdier, and Clair-Antoine Veyrier. 2018. "Voice, power and turntaking in multi-lingual, consecutively interpreted courtroom proceedings with video links." In Here or There: Research on Interpreting via Video Link, edited by R. Skinner, J. Napier and S. Braun, 299-322. Washington: Gallaudet University Press.

Lv, Qianxi, and Junying Liang. 2019. "Is consecutive interpreting easier than simultaneous interpreting? - a corpus-based study of lexical simplification in interpretation." Perspectives 27(1): 91-106. https://doi.org/10.1080/0907676X.2018.1498531

Martin, Anne, and Mustapha Taibi. 2012. "Complexities of high profile interpreting: The case of the Madrid train bomb trial." Interpreting 14(2): 145-164.

Meuleman, Chris, and Fred Van Besien. 2009. "Coping with extreme speech conditions in simultaneous interpreting." Interpreting 11(1): 20-34. 
Mikkelson, Holly. 2010. "Consecutive or simultaneous? An analysis of their use in the judicial setting." Across the Board, Australian Sign Language Interpreters Association 5: 4-7.

Moser-Mercer, Barbara. 1997. "Process models in simultaneous interpretation." Machine translation and translation theory 1(3).

Murphy, Kevin R., and Brett Myors. 1999. "Testing the hypothesis that treatments have negligible effects: Minimum-effect tests in the general linear model." Journal of Applied Psychology 84(2): 234-248. https://doi.org/doi:10.1037/0021-9010.84.2.234

Pöchhacker, Franz. 2004. Introducing interpreting studies. London \& New York: Routledge.

---. 2011a. "Consecutive Interpreting." In The Oxford Handbook of Translation Studies, edited by Kirsten Malmkjær and Kevin Windle, 325-342. Oxford: Oxford University Press.

---. 2011b. "Simultaneous Interpreting." In The Oxford Handbook of Translation Studies, edited by Kirsten Malmkjær and Kevin Windle, 275-293. Oxford: Oxford University Press.

Powell, Martine B., Bronwen Manger, Jacinthe Dion, and Stefanie J. Sharman. 2017. "Professionals' Perspectives about the Challenges of Using Interpreters in Child Sexual Abuse Interviews." Psychiatry, Psychology and Law 24(1): 90-101. https://doi.org/10.1080/13218719.2016.1197815

Razon, Selen, Jasmin Hutchinson, and Gershon Tenenbaum. 2012. "Effort perception." In Measurement in sport and exercise psychology, edited by Gershon Tenenbaum, Robert Eklund and Akihito Kamata, 265-275. Champaign, IL: Human Kinetics.

Rinne, Juha O, Jorma Tommola, Matti Laine, Bernd J Krause, Daniela Schmidt, Valtteri Kaasinen, Mika Teräs, Hannu Sipilä, and Marianna Sunnari. 2000. "The translating brain: cerebral activation patterns during simultaneous interpreting." Neuroscience letters 294(2): 85-88.

Russell, Debra. 2002. Interpreting in legal contexts: Consecutive and simultaneous interpretation. Burtonsville, MD: Sign Media.

---. 2003. "A Comparison of Simultaneous and Consecutive Interpretation in the Courtroom." International Journal of Disability, Community \& Rehabilitation 2(1). http://www.ijdcr.ca/VOL02 01 CAN/articles/russell.shtml.

Russell, Debra, and Kayoko Takeda. 2015. "Consecutive Interpreting." In The Routledge Handbook of Interpreting, edited by Holly Mikkelson and Renee Jourenais, 96-111. Oxon / New York: Routledge.

Seeber, Kilian G. 2015. "Cognitive load in simultaneous interpreting: Measures and methods." In Interdisciplinarity in Translation and Interpreting Process Research, edited by Maureen Ehrensberger-Dow, Susanne Gopfrich and Sharon O'Brien, 18-34. Amsterdam: John Benjamins Publishing Company.

Shaffer, Sarah A., and Jacqueline R. Evans. 2018. "Interpreters in law enforcement contexts: Practices and experiences according to investigators." Applied Cognitive Psychology 32(2): 150-162. https://doi.org/10.1002/acp.3388. https://onlinelibrary.wiley.com/doi/abs/10.1002/acp.3388.

Stern, Ludmila. 2012. "What Can Domestic Courts Learn from International Courts and Tribunals about Good Practice in Interpreting?: From the Australian War Crimes Prosecutions to the International Criminal Court." T\& I Review 2(7-30).

Stern, Ludmila, Uldis Ozolins, and Sandra Hale. 2015. "Inefficiencies of court administration despite participants' goodwill." Journal of Judicial Administration 25(2): 76-95.

Wong, Wan Kei. 2020. "The role of preparation using case-related materials in court interpreting." $\mathrm{PhD}$ Dissertation unpublished, School of Humanities and Languages, Faculty of Arts \& Social Sciences, UNSW. 
Yamada, Hiroko. 2019. "A Direct Application of Simultaneous Interpreting Training without Prior Consecutive Interpreting Work in a University Course." Theory and Practice in Language Studies 9(4): 353-363.

\section{Professor Sandra Hale}

School of Humanities and Languages, Faculty of Arts \& Social Sciences, UNSW Sydney, NSW 2052, Australia

Sandra Hale is Professor of interpreting and translation at UNSW. She is the sole author of The Discourse of Court Interpreting and Community Interpreting, and co-author of Research Methods in Interpreting with Jemina Napier. She is regularly invited to address the judiciary, tribunal members and other legal professionals on how to work effectively with interpreters. She is a NAATI certified interpreter with many years' experience.

Email: S.Hale@unsw.edu.au

ORCID ID: 0000-0003-4291-4022

Professor Jane Goodman-Delahunty

Charles Sturt University, 1 Collins Beach Road, Manly NSW 2095, Australia Jane Goodman-Delahunty, JD, $\mathrm{PhD}$ is an experimental psychologist and lawyer. Her empirical legal studies foster evidence-based decisions to promote social, procedural and distributive justice within organisations and the community. She is a general member of the New South Wales Civil and Administrative Tribunal and a Professor of Legal and Forensic Psychology at Charles Sturt University (Manly Campus).

Email: jdelahunty@csu.edu.au

ORCID ID: 0000-0003-4409-0063

Doctor Natalie Martschuk

Charles Sturt University, 1 Collins Beach Road, Manly NSW 2095, Australia 
Natalie Martschuk, Dipl-Psych, $\mathrm{PhD}$, is an experimental psychologist. Her research interests are procedural justice in legal proceedings, investigative interviewing strategies, jury decision making, and witness reliability and credibility. Further, she has a strong interest in quantitative research methods and meta-analyses. She is a committee member and current Vice President of the Australian and New Zealand Association of Psychiatry, Psychology and Law (ANZAPPL) NSW Branch.

Email: nmartschuk@,csu.edu.au

ORCID ID: 0000-0002-2963-6139

Associate Professor Stephen Doherty

School of Humanities and Languages, Faculty of Arts \& Social Sciences, UNSW Sydney, NSW 2052, Australia

Stephen Doherty is Associate Professor in Linguistics, Interpreting, and Translation, and lead of the HAL Language Processing Research Lab. With a focus on the psychology of language and technology, his research investigates human and machine language processing using natural language processing techniques and combinations of online and offline methods, mainly eye tracking and psychometrics.

Email: $\underline{\text { S.Doherty@unsw.edu.au }}$

ORCID ID: 0000-0003-0887-1049 